\title{
Green Passive Temperature Sensing Technology using Sensitive Ceramic Capacitor
}

\author{
Asma Bakkali \\ LTI Laboratory \\ Abdelmalek Essaadi University \\ B.P.1818, Tangier, Morocco
}

\author{
Youssef Lagmich \\ Polydisplinairy Faculty \\ Hassan $1^{\text {st }}$ University \\ Khouribga, Morocco
}

\author{
Abdelouahid Lyhyaoui \\ LTI Laboratory \\ Abdelmalek Essaadi University \\ B.P.1818, Tangier, Morocco
}

\begin{abstract}
Acquiring temperature measurements using conventional methods can be costly and technically challenging. These active temperature sensors limit the environmental conditions in which they can be deployed. Most of them cannot tolerate wide temperature ranges and they are susceptible to failure in high radiation environments. This paper presents an energy autonomous wireless temperature sensing system without requiring physical contact or active elements. The environmental friendly device is made up of a planar capacitor integrated into passive filter. As the temperature changes, the capacitance is modified and consequently alters the resonant frequency of the circuit. The sensor can be integrated with an antenna and interrogated at distance by a reader. To prove this concept, a wireless sensing system is designed and simulated using a variable capacitor. Simulations were performed in order to optimize the device design and to verify the frequency shift with sensitivity of $2.85 \mathrm{MHz} /{ }^{\circ} \mathrm{C}$. This design allows wireless temperature sensing, thus making it an effective solution for distant temperature monitoring applications. However, this developed technique is general enough to be implemented to high temperature application by using other capacitors made of high temperature ceramics sensitive materials.
\end{abstract}

\section{Keywords}

Battery free, Passive, Sensitive capacitor, Resonant frequency, Temperature sensor, Wireless.

\section{INTRODUCTION}

Fueled by the demand of smart sensors for environmental and industrial applications, significant amount of research has been performed on passive wireless sensors due to their several advantages over other competing sensing systems. These advantages include battery free operation, low maintenance cost, higher reliability and a better chance of toleration in high temperature environments. Although the most active sensing technologies offer outstanding performances in terms of resolution and time response, they require physical connection, battery power supply or expensive equipments for acquiring environmental variables information. These limitations make these existing active sensing technologies unsuitable for most monitoring applications [1].

Because energy becomes one of the major concerns, solutions based on active sensors have been ignored to give way to passive techniques. Therefore, significant amount of research have made contributions to advance passive wireless sensing technologies and extend their applications in many areas, such as pressure sensor, humidity sensor, and temperature sensors [2], [3], [4], [5], [6]. In this context, many papers have been published related to passive technologies, removing contacts, active elements, or power supplies to be contained within the sensor.

Among various passive sensing techniques, those based on thermocouple technology have been developed extensively in the past decade. Most of available high temperature thermocouples are able to measure temperature up to $2300^{\circ} \mathrm{C}$. However, this technology requires sophisticated slip ring mechanisms to ensure the continuity of electrical contacts while the signal outputting is weak and can easily be affected by common mode noises under high temperature environments [7], [8], [9]. Surface Acoustic Wave (SAW) technology were regarded as one of the most promising passive sensing techniques. SAW devices were used for various sensing applications as temperature, pressure and humidity monitoring. The principle of using SAW sensors is based on involving electrically acoustic waves and then reconverting the energy of the transduced wave back into an electrical signal for temperature measurement. However, they still suffer from some weaknesses including low sensitivity, calibration difficulties, and the RF interrogation signal that has to cover twice the distance between the reader and the sensor unit. Therefore, these devices cannot be implemented in some interesting applications, such as temperature sensing in turbine engines [10], [11], [12]. The Radio Frequency IDentification (RFID) technology has been another attractive passive solution developed in order to overcome the energy consumption reduction needs. The RFID devices are able to sense, power themselves, and communicate. While they have no power source onboard, their lifetime is virtually unlimited. Because, they cannot be interrogated at a range of more than 1 $\mathrm{m}$, the main drawback of the passive RFID technology is the limited reading distance [13], [14], [15].

Previous work by Y. Jia et al at Puerto Rico University has demonstrated very attractive results based on a passive LC resonant telemetry scheme, relying on a frequency variation output in order to measure temperatures, without need for physical contacts, active elements, or power supplies [16], [17], [18]. However, technology used for this passive sensing device suffers from some drawbacks in terms of operating frequency and reading distance. This project focuses on making the potential of this emerging technology a reality. 


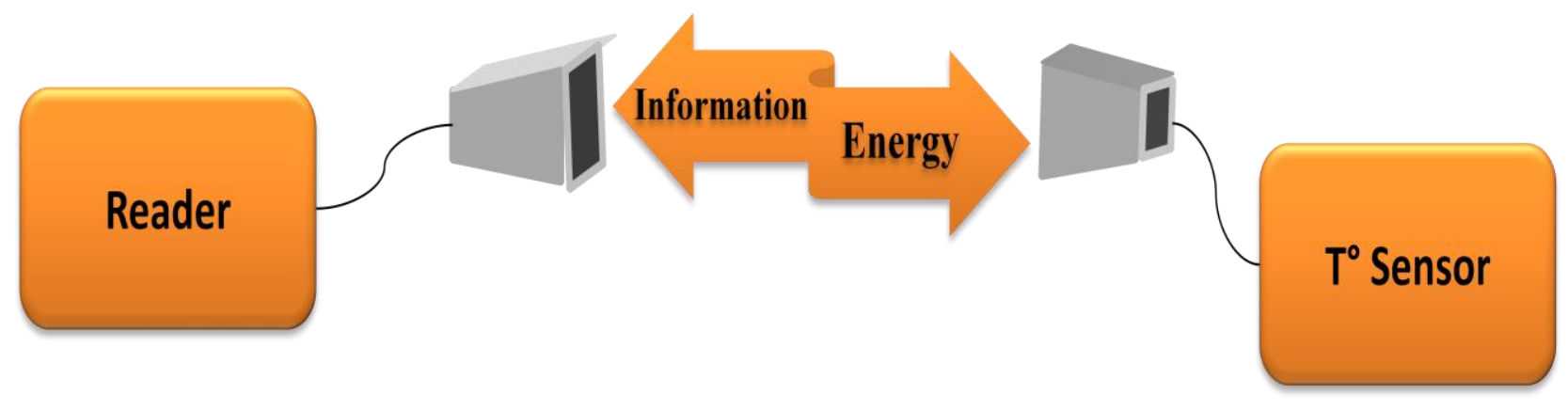

Fig 1: Schematic of the Wireless Proposed Communication

Nowadays, temperature passive sensors appear as a key technological solution for various applications, requiring energetic autonomy, small sized and stable sensing devices. The challenges of developing these sensors include high accuracy, long term measurement stability and survive harsh working environments. As a result, innovative and alternative sensing techniques are needed to respond to the requirements for passive sensing applications.

This paper is intended to propose a wireless battery free temperature sensor. The temperature variations can be translated into a resonant frequency using a sensitive capacitor. Therefore, the device resonant frequency is related to temperature properties. By measuring this frequency using wireless technique, the information regarding temperature variation can be retrieved. This research is referred to extend the knowledge base of passive temperature sensing technologies in high temperature conditions and advance environment monitoring technologies.

\section{PRINCIPLE AND DESIGN OF THE PROPOSED TEMPERATURE SENSOR}

The wireless interrogation and remote powering is achieved through a remote reader using an electromagnetic link between the reader's and the sensor's antennas. Temperature change is wirelessly translated into a frequency shift, therefore, this information concerning the resonant frequency is also transmitted to the reader side through this link.
As illustrated in Fig.1. The temperature information is detected by the reader across its antenna and then transmitted to a computer in order to analyze and store the data. The capacitance of the sensor changes in response to the variable temperature. Therefore, the resonant frequency of the device changes and the remote reader can detect frequency variations in the sensor's response across its antenna.

The temperature sensor, as illustrated in Fig.2, is designed and optimized using Multisim software. The proposed device is composed of variable capacitor for wireless temperature monitoring integrated with passive bandwidth filter. This planar capacitor is the basic design principle of this sensing system. Besides, its structure makes the sensor easy to attach and can be used for a wide temperature monitoring applications.

In high temperature environments, the value of the capacitance changes linearly with the temperature variation as seen in Fig.3. This rising level of the capacitance within the temperature modifies the resonant frequency of the device.

The core of the capacitor is made of a sensitive ceramic material that presents excellent dielectric properties with a temperature dependent dielectric constant value, and it is able to tolerate high temperatures up to the $200^{\circ} \mathrm{C}$ in which conventional materials cannot survive. The ceramic material is covered with a conductive layer of $\mathrm{NiCr}$, allowing its work as a capacitive sensing element. 


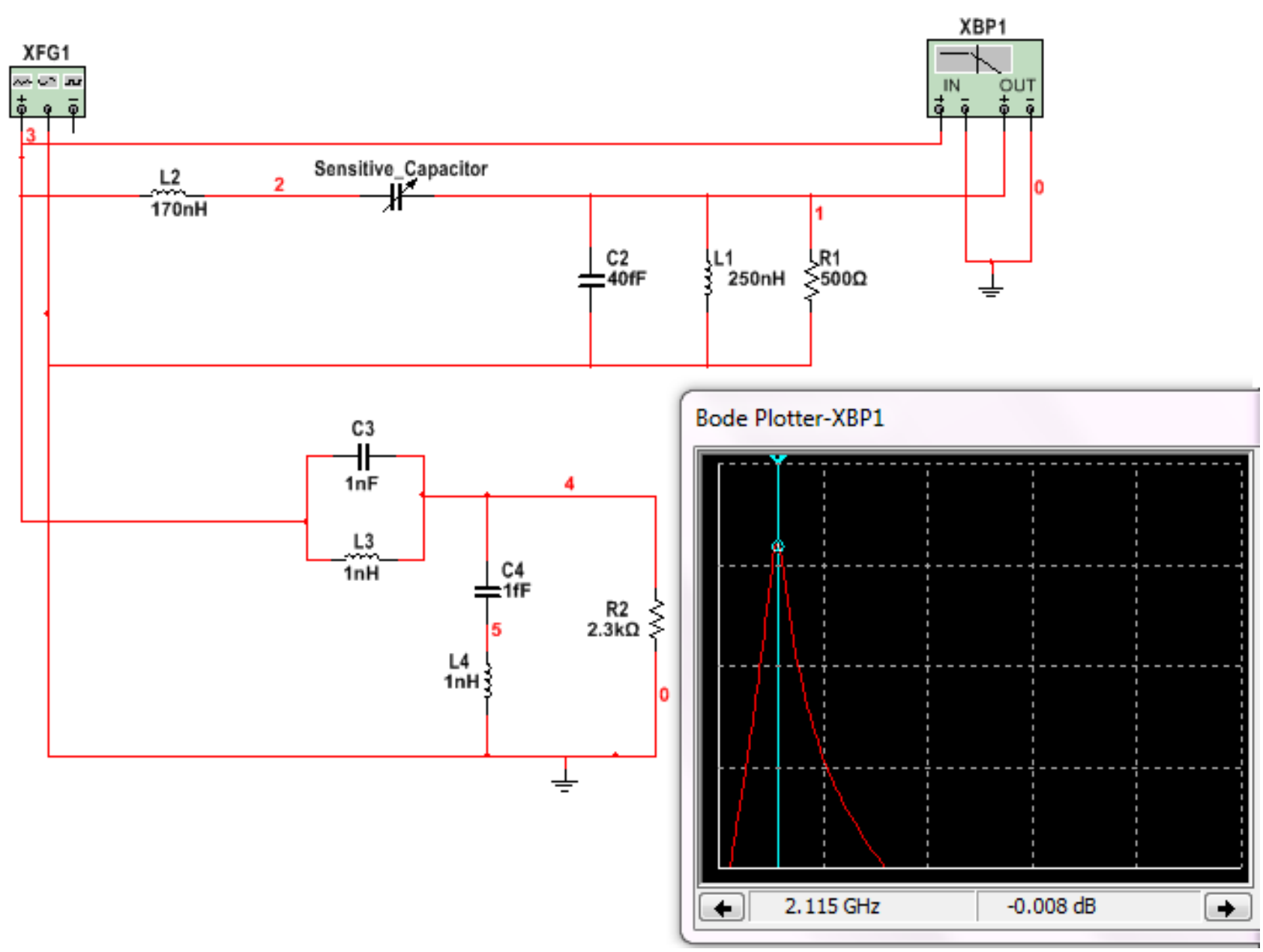

Fig 2: Proposed Temperature Sensing System

The sensor capacitance is a function of the dielectric constant of the Lead-Lanthanum-Zirconate-Titanate sensitive material as demonstrated in equation 1 .

$$
C(T)=\varepsilon_{o} \varepsilon r(T) . \frac{s}{\mathrm{e}}
$$

Where,

$\varepsilon_{0}$ : Permittivity of free space, $8.85 \times 10^{-12} \mathrm{~F} / \mathrm{m}$

$\varepsilon_{\mathrm{r}}$ : Temperature dependent dielectric material permittivity

S: Area of the electrode plate

e: Thickness of dielectric material and distance between the electrode plates

Once the planar capacitor is exposed to variable temperatures, the dielectric constant has a linear variation with the temperature.

As previously shown, the temperature sensor has a simple design consisting of the sensitive capacitor and a passive filter. The filter choice is based on the many advantages that it offers in terms of $\mathrm{Q}$ factor, sensitivity and frequency operation. This device consists, in addition to the variable capacitor, of three static capacitors and four inductors. In addition to two resistors, losses representatives are added.

The ceramic multi-layer capacitor is integrated into the passive RLC circuit as a frequency controlling element: The capacitance changes due to temperature variation are translated into frequency shift.

\section{SIMULATIONS RESULTS:}

The simulation has been performed to offer a general idea of relationship between the resonant frequency and dielectric properties capacitor, as demonstrated in Fig.4.

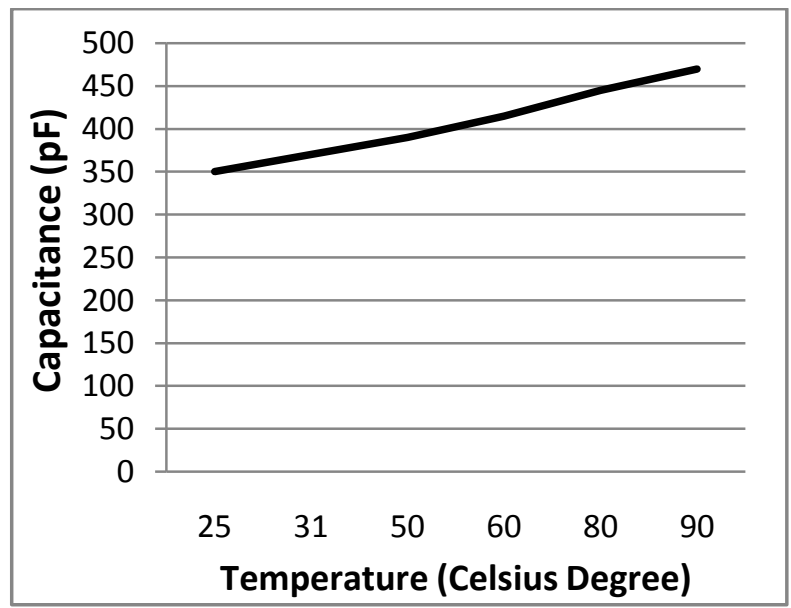

Fig 3: Capacitance vs. Temperature Plot 
Since the dielectric properties of the capacitor are temperature dependent, a shift in the sensor's resonant frequency is produced in response to temperature changes. A linear dependency of the resonant frequency on the capacitance is clearly seen.

As previously described, there is a temperature dependency on the material dielectric constant, resulting in a change of the sensor capacitance. Figure 5 illustrates the variation of the resonant frequency due to variations in temperature. As a result, a linear variation between frequency and temperature is noticed. Therefore, the device resonant frequency changes versus temperature properties. This variation information can be captured wirelessly by the reader, and the antenna used in this system should cover the range of possible resonant frequencies.

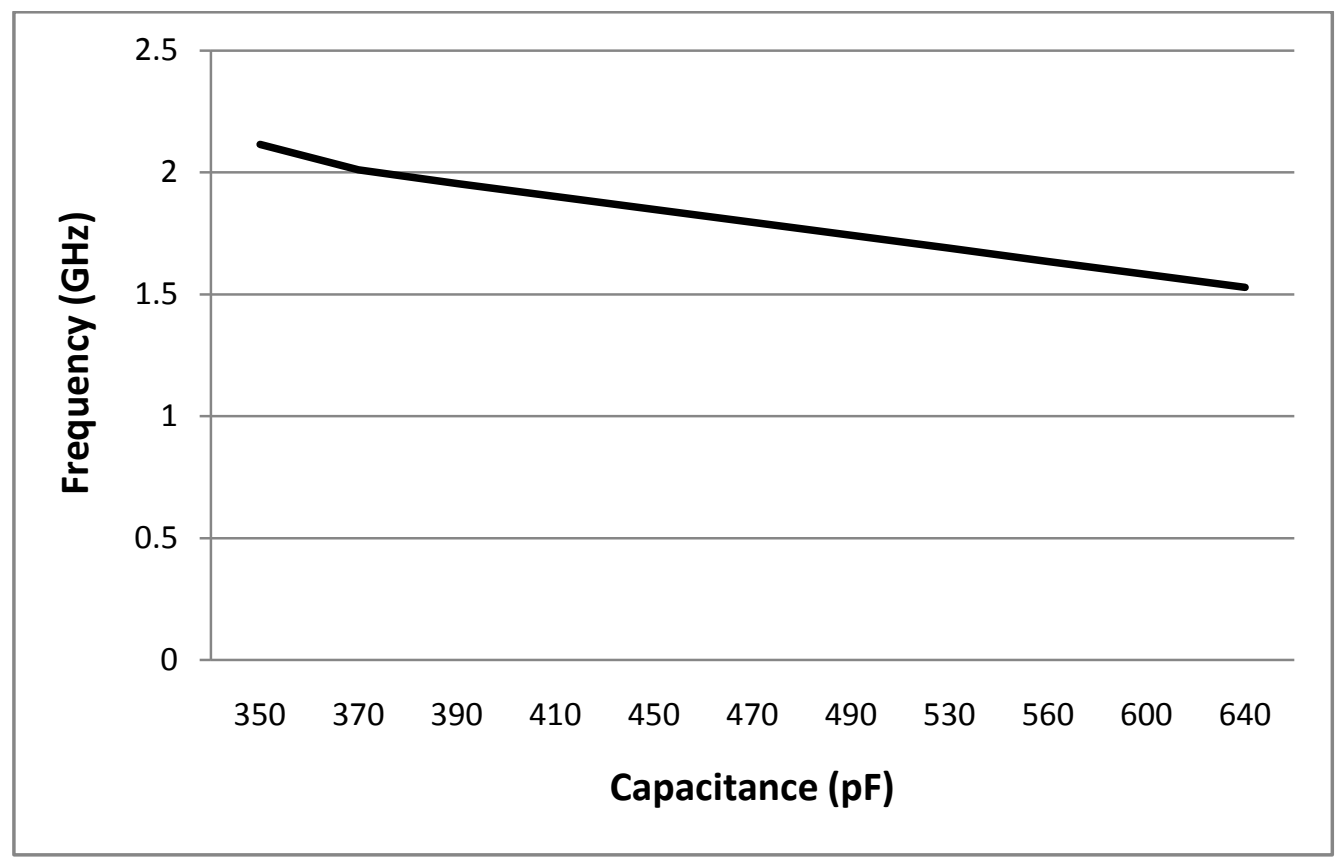

Fig 4: Sensor prototype temperature response; Sensor Capacitance vs. Frequency

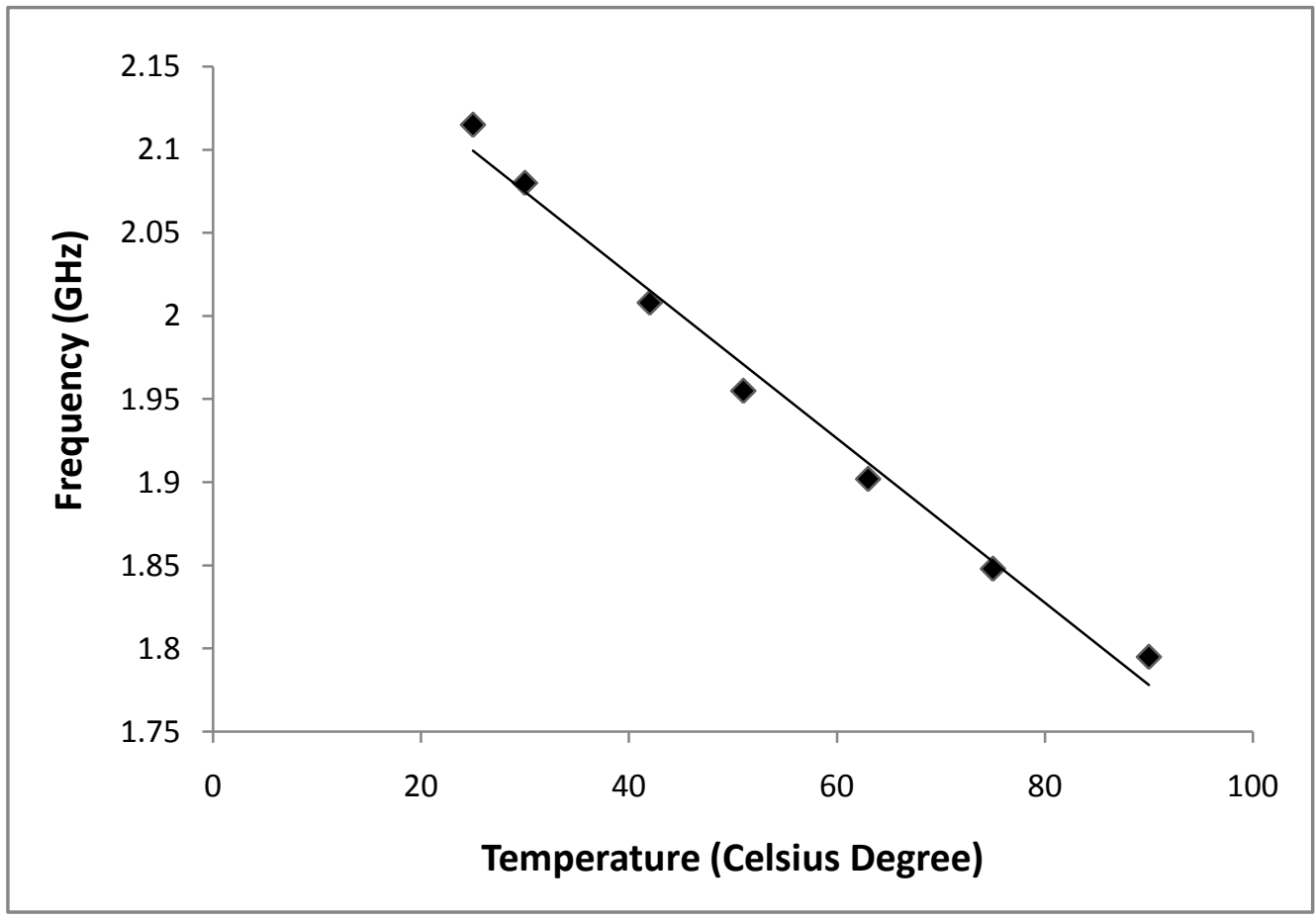

Fig 5: Frequency vs. Temperature Plot 
Results simulations demonstrate numerous benefits of the proposed device compared with others routinely used. Based on the excellent features of the passive filter as well as the good sensitivity of the ceramic capacitor, this autonomous sensor presents a promising solution over existing temperature sensors: efficiency in passive remote measurement of temperature, independence from onboard power, high frequency operation, flexibility of integration in sensors networks and low investment and implementation cost.

Therefore, the device would be a powerful tool for many interesting applications since it offers very low power consumption and provides environmentally friendly temperature measurement.

\section{CONCLUSION}

New trends are moving in the direction of wireless and self powered sensing system. A passive sensor device reducing the problem of environmental impact and based on variable planar capacitor has been successfully demonstrated in this project. The temperature sensitive capacitor has been integrated into the RLC resonant circuit to create a frequency sensing mechanism, able to increase dramatically sensitivity and time response of the wireless temperature sensors. The sensed resonant frequency has a noticeable response versus temperature changes.

As a result, the temperature sensing technology presented in this paper offers many advantages including wireless interrogation, battery free and a simple design. Future work will be performed in order to optimize and extend the interrogation distance and the operating temperature range. Further investigation is also under way to verify the correlation between the conceptual and experimental results, and provide an equivalent device since it is a high frequency system.

\section{REFERENCES}

[1] Kar, K., Krishnamurthy, A., and Jaggi, N. 2005. Dynamic Node Activation in Networks of Rechargeable Sensors.

[2] Sardini, E., and Serpelloni, M. 2009. Passive and SelfPowered Autonomous Sensors for Remote Measurements.

[3] Pons, P., Aubert, H., Menini, P., and Tentzeris, M. 2011. Wireless Passive Autonomous Sensors with Electromagnetic Transduction.
[4] Jatlaoui, M.M., Pons, P., and Aubert, H. 2008. Pressure Micro-sensor based on Radio- Frequency Transducer

[5] Hallil, H., Menini, P., and Aubert, H. 2009. Novel millimeter-wave gas sensor using dielectric resonator with sensitive layer on $\mathrm{TiO}_{2}$.

[6] Hallil, H., Menini, P., and Aubert, H. 2009. Novel microwave gas sensor using dielectric resonator with $\mathrm{SnO} 2$ Sensitive Layer.

[7] Bajzek, T.J. 2005. Thermocouples: a sensor for measuring temperature

[8] Ming, Z. 2010. Research and Implement of Thermocouple Sensor and Microcontroller Interface

[9] Zhang, P., Ouyang, G., and Tong, Y. 2010. Thermocouple sensor technology applied in detecting thermal flaw inner cylinder head.

[10] Bao, X. Q., Burghard, W., Varadan, V. V., and Varadan, K. V. 1987. SAW Temperature Sensor and Remote Reading System.

[11] Durdag, K. 2009. Wireless Surface Acoustic Wave Sensors.

[12] Pohl, A. 2000. Review of Wireless SAW Sensors.

[13] Yeager, D. J., Sample, A. P., and Smith, J. R. 2008. Wisp: A passively powered UHF RFID tag with sensing and computation

[14] Sample, A. P., Yeager, D. J., Powledge, P. S., and Smith, J. R. 2008. Design of an RFID-based battery-free programmable sensing platform.

[15] Buettner, M., Greenstein, B., Sample, A., and Smith, J. R. Revisiting Smart Dust with RFID Sensor Networks. University of Washington, Intel Research Seattle.

[16] Wang, Y., Jia, Y., Chen, Q., and Yanyun, W. 2008. A passive wireless temperature sensor for harsh environment applications

[17] Jia, Y. 2009. Passive Wireless Temperature Sensors and Pressure Sensors. Laboratory for Integrated Sensing Technologies. University of Puerto Rico.

[18] Rodriguez, R.I., and Jia, Y. 2011. A wireless inductivecapacitive (L-C) sensor for rotating component temperature monitoring. 